\title{
Contents, Vol. 151, No. 1-3, 1966
}

Inhaltsverzeichnis - Contents - Sommaire Hauptreferate - Main Lectures - Rapports principaux Radnót, M. und Gall, J. (Budapest): Tumoren des Tränen-

sackes

Discussion

Dollfus, M. A. (Paris): Epithéliomas palpébraux. Etude clinique

et thérapeutique

Discussion

Hewson, G. E. (Galway): Scleral Tumours

Rintelen, F. (Basel): Zur Histopathologie der Lidtumoren . .58

Discussion

Thíel, R. (Frankfurt a. M.): Dis bösartigen Geschwülste der

Bindehaut

68

Discussion 73

Pãcuraríu, I. (Cluj): Les tumeurs malignes de la cornée ... 75

Discussion 93

Ashton, N. and Wybar, K. (London): Primary Tumours of the

Iris

Discussion

Ill

Francois, J. (Gand): Diagnostic des mélanomes malins de la

choroïde 114

Francois, J. et Hanssens, M. (Gand): Histopathologie des mélanomes malins de la choroïde 135

Discussion 155

Char amis, J. et Topalís , C. (Athènes): Traitement des mélanomes malins de la ch oroïde 161

Discussion 176

Sezer, N. and Barishak, JR. (Istanbul): The Diagnosis of Retinoblastomas 184 IV

Inhaltsverzeichnis

Schappert-Kímmíjser, J.; Hemmes, G. D. and Níjland, R. (Den Haag): The Heredity of Retinoblastoma

Discussion

212

Stallard, H. B. (London): The Treatment of Retinoblastoma 214

Discussion 230

Schwab, F. (Wien): Die Tumoren der Netzhaut und der Ader-haut mit Ausnahme des Retinoblastoms und des bösartigen

Melanoms der Aderhaut 231

Arkhangelsky, V. N. (Moscow): Neoplasms of the Optic Nerve 260

Casanovas, J. (Barcelone): Tumeurs métastatiques de Гæil et 
del'orbite $\quad 272$

Discussion 282

Ehlers, H. (Kopenhagen): Die Augensymptome bei der Reck-

linghausenschen Neurofibromatose 284

Discussion 301

Bertelsen, T. I. (Bergen): Diagnostische Methode bei einsei-

tigem Exophthalmus

309

Discussion

330

Back, J. und Feyrter, F. (Wien): Die Tumoren der Tränendrüse 331

Moro, F. (Padoue): Les pseudotumeurs de Гorbite 349

Stankovic, I.; Lítrícin, 0. et Stefanovíc, P. (Belgrade): Incidence des tumeurs voisinage 390

Discussion 402

Babel, J. (Geneve): Les malformations pseudotumorales du

globe oculaire

405

laires

427 D. Discussion

426 